\title{
RELEVANT ARITHMETIC AND Mathematical Pluralism
}

\author{
Zach Weber
}

University of Otago

\begin{abstract}
In The Consistency of Arithmetic and elsewhere, Meyer claims to "repeal" Gödel's second incompleteness theorem. In this paper, I review his argument, and then consider two ways of understanding it: from the perspective of mathematical pluralism and monism, respectively. Is relevant arithmetic just another legitimate practice among many, or is it a rival of its classical counterpart - a corrective to Gödel, setting us back on the path to the (One) True Arithmetic? To help answer, I sketch a few worked examples from relevant mathematics, to see what a non-classical (re)formulation of mathematics might look like in practice. I conclude that, while it is unlikely that relevant arithmetic describes past and present mathematical practice, and so might be most acceptable as a pluralist enterprise, it may yet prescribe a more monistic future venture.
\end{abstract}

\section{Introduction}

Circa 1976, Robert K. Meyer ${ }^{1}$ made a very bold claim. He claimed to have done nothing less than overturn - "repeal" - Gödel's second incompleteness

\footnotetext{
${ }^{1}$ I met Meyer once, when I was a PhD student, shortly before he died. He asked me what I was working on. I said "Set theory formulated relevantly." He said, "That's a bad idea. That won't work." Then he gave a very charming talk about relevant logic as the key to the universe. His work and influence have been hugely important to me and it is an honor to contribute to this volume.
}

Australasian Journal of Logic (18:5) 2021, Article no. 14 
theorem of $1931 .^{2}$ In this paper I will consider some ways to make sense of Meyer's claim.

Of course, Meyer is far from alone in purporting to disprove (in some sense) a proven theorem; nor is he alone in making such claims in lengthy, selfpublished typescripts circulated (only) privately. I find such announcements waiting in my in-box once or twice a year. ${ }^{3}$ But I've learned not to respond to those messages, and I certainly don't usually write entire papers responding to such work. Yet here I do respond to Meyer, because his claim-that arithmetic suitably formulated evades Gödel's second theorem-is different than those other typescripts: whereas mathematical crankery inevitably falls apart upon closer inspection, The Consistency of Arithmetic does not. ${ }^{4}$ It involves a coherent mathematical result, and leaves us with the challenge of how philosophically to understand what it means.

After reviewing his argument, I will present two ways of interpreting Meyer's result, a pluralist interpretation and a monist interpretation. Roughly, a pluralist will say that Meyer's result is correct but without being rivalrous to other, incompatible results: for any logic $L$, there is arithmetic-in- $L$, and what Meyer showed is that Gödel's theorem does not hold for some particular choice of $L$, which does nothing to impugn the better-known results about some other choice of $L$. A thousand flowers bloom etc. ${ }^{5}$

A monist, on the other hand, might say that arithmetic (taken absolutely, without relativisation to some logic) either can prove its own consistency, or it cannot; Meyer was talking about absolute or True Arithmetic; and so either Meyer was correct, or he was not. If True Arithmetic is classical, then Meyer was simply wrong, or at least misrepresenting his results (because [18] is really about something other than arithmetic). If True Arithmetic is relevantor more generally, paraconsistent - then maybe Meyer was correct, and the reports of the death of Hilbert's program have been greatly exaggerated.

2 "That theorem (or at least the significance usually claimed for it) was a mistake," Meyer says in [18]. He puts it in various ways in various papers. The proof "escapes incautious formulations" [23, p. 917]; it "removes some of the sting" [21, p. 247]. That 1996 paper is a precis of Meyer's 1970s work, but since it was published 20 years later, I take it that Meyer never retracted his claims, even after the negative results in [11]; indeed, the 1996 paper says that "Gödel's theorems are dirty tricks" (boldfacing in the original).

${ }^{3}$ See $[14]$.

${ }^{4}$ I will be primarily focused on [18], though I will also have [17] on hand as well as a raft of other related works by Meyer.

${ }^{5}$ This is to echo e.g. Shapiro in [39], not Chairman Mao. (Thanks to a referee here.)

Australasian Journal of Logic (18:5) 2021, Article no. 14 
In presenting these ways of understanding Meyer, I will look at a few new worked examples from relevant arithmetic and substructural mathematics, to give us more help in clarifying, or maybe just muddying, the issue. I will conclude by distinguishing between two projects, one descriptive, the other normative, and apply the pluralist/monist distinction to them, to try to come to grips with Meyer's work and its legacy.

\section{The Consistency of Arithmetic}

Hilbert didn't know, but we all do, that arithmetic cannot prove its own consistency, by Gödel's second theorem. Or can it? Meyer challenges the accepted Gödelian facts, essentially on the basis that Peano, Russell, Hilbert, Gödel et al were using the wrong logic to formalize arithmetic, and that arithmetic formulated using a better logic will do better. Gödel's theorems are "puzzles about the logical superstructure of arithmetic, which do not infect the properly arithmetic" [18, p. 9]. Meyer's case can be given in two steps, a critical argument and positive evidence. Unlike Meyer, I give them in reverse order.

\subsection{The positive case}

I think it is fair to say that, whatever doubts or complaints one has about Gödel's news of 1931, those opinions are of much more interest if they come with some constructive alternative suggestions. Meyer can mount a case that classical arithemtic has got it wrong because he has a clear and cogent rival system that, he says, gets it right.

The systems in question, of course, are classical Peano Arithmetic PA, and relevant arithmetic $R^{\sharp}$. To obtain $R^{\sharp}$, one takes PA and replaces the underlying classical logic with the first order relevant logic $R$ (so the extensional connectives $\wedge, \vee, \neg$ and quantifiers are now those of $R$ ). Then one replaces the occurrences of the material conditional $\supset$ in the Peano axioms with relevant implication $\rightarrow$. (Mostly. Mathematical induction is formulated as a rule, rather than as an axiom, "in a self-explanatory meta-linguistic way" [17, p. 14].) The set of theorems is then the closure of the axioms under logical consequence (as given by $R$ ). The details are a little more involved, but that's the idea; see $[17$, p. 13, 14].

Now, if we assume that $R^{\sharp}$ is at least a plausible candidate for being a

Australasian Journal of Logic (18:5) 2021, Article no. 14 
formalization of arithmetic, then Hilbert's question for it would be: "Okay, well is this system reliable, and if so, can it confirm its own reliability?" 6 To show basic reliability, semantically-minded logicians ask to see a model of $R^{\sharp}$. Ideally, this would be the abstract structure $R^{\sharp}$ is describing, intended to be the natural numbers $\mathbb{N}$; but, much like its first order classical counterpart, $R^{\sharp}$ does not pin down only the desired omega chain of $\mathbb{N}$; cf. [24]. So, failing a categorical interpretation, we will settle for any non-trivial model which $R^{\sharp}$ is sound with respect to: a structure that at least satisfies every theorem of $R^{\sharp}$, and which at most leaves at least one (false) sentence of $R^{\sharp}$ unsatisfied. The key discovery from this period of the development of inconsistent mathematics is that this can be had very easily, as follows.

$R^{\sharp}$ would appear to be describing an infinite structure - but it may be soundly interpreted in the positive integers modulo two. That is, take $\mathbf{s}$ to be the successor function, and $\{0,1\}$ as the domain of interpretation, with $\mathbf{s}(0)=1$ and $\mathbf{s}(1)=0$. If we use a three-valued $\operatorname{logic}^{7}$ to interpret the sentences $0=0$ and $1=1$ as both taking the value $\mathrm{b}$ (true (but not only true)), then "it is readily observed that" all the true sentences of $R^{\sharp}$ take designated (i.e. 'at least true') values. But by construction, the sentence $0=1$ is assigned the value $\mathrm{f}$ (false), an undesignated value. So, once the details are filled in, we have a model that satisfies $R^{\sharp}$ but without satisfying $0=1$. This shows that, at the least, $R^{\sharp}$ is absolutely consistent, or as we say more usually, non-trivial. Cf. [28, ch. 1].

Hilbert asked for a finitary proof of the reliability of arithmetic, a demonstration by elementary and ordinary means. But what could be more finitary than a model of arithmetic that looks like this:

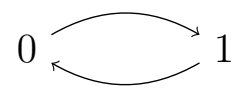

\footnotetext{
${ }^{6}$ As I write that now, and after reading Meyer's papers, the first part of Hilbert's question seems eminently reasonable, but the second part looks odd. If the system in question is arithmetic, why are we expecting it "to be muttering introspectively about itself at all" [18, p. 3]? But nevermind.

${ }^{7}$ Since we are only looking for soundness in a model, not completeness, the task of dealing with the logic is simplified too; cf. [23]. The conditional for $R$ does not have a truth table, but the nearby three valued logic RM3 does, with truth values true (t), false (f), and both (b), making RM3 "the laboratory of relevant logic" according to Meyer (according to $[9$, p. 81]). RM3 is Priest's logic $L P$ with a better conditional attached [33, p. 125].
}

Australasian Journal of Logic (18:5) 2021, Article no. 14 
? So $R^{\sharp}$ has a finitary consistency proof - an argument that it is coherent, that anyone who can count to two should be able to recognize as legitimate. ${ }^{8}$ Of course, absolute consistency - non-triviality - is proven at the cost of simple consistency: the induced interpretation says that both $1+1=0$ and $1+1 \neq 0$ are true, for instance. Meyer does not establish that there is a finitary consistency proof in the sense that at least one of $A, \neg A$ is unprovable for all $A$, or of the system showing its own soundness (all theorems are true); these are just as out of reach as ever. ${ }^{9}$ Indeed, we've just seen that there are models of $R^{\sharp}$ where some $A, \neg A$ are both satisfied, the so-called inconsistent models of arithmetic (so called even though they are completely consistent models of inconsistent theories). But, Meyer suggests, one should not get too worked up over these sorts of inconsistencies; they represent low probability, low cost, contingencies.

We do not think of such negated formulas as $2+2 \neq 4$ as true. But the point is, even if we did, it still wouldn't follow that $2+2=5$. That's the idea, anyway [Meyer and Urbas 1986].

So we have a proof that $R^{\sharp}$ is not completely unreliable, because it may be satisfied by some structure, and not only that, it is paraconsistent. To people of discerning taste, this tastes like good news. Because PA "fails to account for relevance as a constituent in valid arithmetical arguments," says Meyer, it is "unable to discriminate between logical anomaly and total arithmetical breakdown" [18, p. 9]; not so for PA's relevant rival.

That's where Meyer gets the nerve to challenge Gödel's theorem. What does he say against it?

\subsection{The critical case}

The positive part of the case, just canvassed, makes clear that $R^{\sharp}$ is more than just PA with a better entailment connective. What is really doing the work is not, in fact, much about $\rightarrow$ at all but rather negation, and the fact

\footnotetext{
${ }^{8}$ Meyer says the proof "will be recognized as correct by anyone who can count to 3 " $[18$, p. 1] but this unduly excludes people who cannot count that high.

${ }^{9}$ Priest has taken the matter further with the idea that 'naive' arithmetic can prove its own soundness [32, ch. 17]: since the argument for soundness is a simple induction, "if something is naively proved then this fact itself constitutes a proof that $A$ is provable" [32, p. 238].
}

Australasian Journal of Logic (18:5) 2021, Article no. 14 
that negation in $R$ is paraconsistent. That's why $R^{\sharp}$ can have a finite model: there can be a lot of inconsistent noise without total collapse. ${ }^{10}$ The difference between formalizations of arithmetic turns on negation. Meyer argues that classical negation is not negation, by the following argument.

Whether arithmetic can prove its own consistency, or not, is a matter of whether or not it can prove some sentence CON that expresses 'arithmetic is consistent'. Any statement of consistency will involve the particle 'not' in some respect. E.g. CON may be the sentence 'It is not provable that $A$ and not $A$ ' or 'If $A$ is provable then not- $A$ is not provable'. If the language of arithmetic does not include a connective that faithfully expresses 'not', then it cannot even express a consistency statement CON. Failing to prove some sentence that inadequately expresses consistency does not count as failing to prove consistency. "No one expects us to prove what we cannot say" [18, p. 4]. So, if there is evidence that the particle representing 'not' in classical logic is not not, then can it express the genuine consistency of arithmetic? It cannot. Here is Meyer's evidence (from [18, p. 3], [21, p. 3]).

Let $\operatorname{Prov}(x)$ be a provability predicate, an expression definable within arithmetic meaning that $x$ is provable in arithmetic. Let $\ulcorner$.$\urcorner be a coding$ device. Let $\vdash A$ be read as ' $A$ is a theorem of arithmetic'. Well, then

$$
\operatorname{Prov}(\ulcorner A\urcorner) \text { iff } \vdash A
$$

And any connective in the language of arithmetic that faithfully represents 'not' would, says Meyer, have to validate

$$
\neg \operatorname{Prov}(\ulcorner A\urcorner) \text { iff not } \vdash A
$$

because the ' $\neg$ ' on the left is meant to be capturing the 'not' on the right. But not just this; Prov is a provability predicate in arithmetic, i.e. Gödel showed

$$
\vdash \operatorname{Prov}(\ulcorner A\urcorner) \text { iff } \vdash A
$$

And yet the connective that represents 'not' in classical arithmetic does not validate

$$
\vdash \neg \operatorname{Prov}(\ulcorner A\urcorner) \text { iff not } \vdash A
$$

\footnotetext{
${ }^{10}$ And that's why Priest has been able to carry off many of the same results for his paraconsistent arithmetic formulated in just the extensional logic LP [30, 31], [32, ch. 17]and that's why the potential for Meyer's contribution goes beyond just relevant logic, but for paraconsistent logic more generally.
}

Australasian Journal of Logic (18:5) 2021, Article no. 14 
("on pain of total bankruptcy otherwise of standard mythology"). For example, from the Gödel sentence $\mathfrak{g}$ that says $\neg \operatorname{Prov}\ulcorner\mathfrak{g}\urcorner$, if PA is consistent then it is not the case that $\vdash \mathfrak{g}$, but it is also not the case that $\vdash \neg \operatorname{Prov}(\ulcorner\mathfrak{g}\urcorner)$. Therefore, the connective that represents 'not' in classical arithmetic does not faithfully express 'not' and so the failure to prove some expression CON in terms of this not-really-not is not a concern.

[T] he little sign ' $\sim$ ' is supposed to mean 'not'. The real import of Gödel's arguments may be summed up succinctly thus: ' ' never means what it is supposed to mean, within a particular sufficiently strong system intended seriously to formalize mathematics [21, p. 248].

What Meyer seems to be taking issue with is the failure of classical negation to commute with $\vdash$; from

Exclusive If $\vdash \neg \operatorname{Prov}(\ulcorner A\urcorner)$ then not $\vdash \operatorname{Prov}(\ulcorner A\urcorner)$

Exhaustive If not $\vdash \operatorname{Prov}(\ulcorner A\urcorner)$ then $\vdash \neg \operatorname{Prov}(\ulcorner A\urcorner)$

Meyer seems to expect that any 'natural' arithmetic should validate at least the second, exhaustion, as an adequacy condition for formalizing 'not'. For what it's worth, I think that both of these directions are highly desirable and intuitive, and dropping either ends up suggesting that our (meta)linguisitc 'not' is not fully captured by (the object level) ' $\neg$ '. (A similar issue arises in discussion of a truth predicate.) Still, this is not too far away from simply insisting that, as above, any decent formalization of arithmetic should not succumb to Gödel's theorems, so it isn't likely to preach to the unconverted. Rather what we should just say, with Meyer, is that our 'not' is not mysticism or irrational noise, and so it should have a precise formulation; and if we want arithmetic to prove a sentence CON that really means 'consistency' then formulation of $\neg$ in arithmetic should really mean 'not'. Everyone agrees that what shows up in PA is, in some sense not the whole truth - that's what Gödel proved - so it's then a matter of deciding whether to expect the precise formulation of genuine negation to show up exhaustively in arithmetic. Or not.

Rather than answer that question, I would like to stop and investigate what we are even asking when we ask that question. If we are asking "Is this or that formalization getting it right?" what do we mean?

Australasian Journal of Logic (18:5) 2021, Article no. 14 


\section{Mathematical Pluralism and Monism}

\subsection{A Pluralist View}

Meyer claims that he is repealing Gödel's second theorem. Many readers, I suspect, would be inclined to say that this claim is misleading. For Gödel's second theorem, as we all know, says that classical PA cannot prove its own consistency; but this is not what Meyer challenges. Rather, he intends to show that a different system, relevant arithmetic $R^{\sharp}$, can prove its own (absolute) consistency. So would it not have been more transparent for Meyer to phrase his results as saying that Gödel's second theorem does hold of some formalizations of arithmetic and not others? For indeed, this seems the likely way that his result is understood. And one way of understanding that is via mathematical pluralism. ${ }^{11}$

Like logical pluralism, there are different accounts of mathematical pluralism. Like logical pluralism, they all circle around the idea that different and apparently rival mathematics may be in fact non-rivalrous, and are at least equally legitimate qua mathematics.

One can be a mathematical pluralist without straying outside of conventional mathematics. One may just think of questions like 'do parallel lines ever meet?' or 'how many points are there on a line?' and observe that there are multiple independently consistent models (of geometry, of set theory) that yield different answers. According to Hamkins [13], for example, there just is no one answer to the question 'is the power of the continuum $\aleph_{1}$ ?' There isn't a single universe of mathematical truths but rather a multiverse, and that's okay. There are many interesting structures to explore, even just within classical models of ZFC. An even more open-minded version of mathematical pluralism is an ecumenical view akin to Carnapianism about logical tolerance (cf. [36]): that all abstract structure is, qua structure, equally legitimate.

An apparently less moderate version of pluralism recognizes various unconventional mathematical practices, using non-classical logic, such as smooth infinitesimal analysis, or paraconsistent set theory [39]. In smooth infinitesimal analysis, where the law of excluded middle fails, all functions are continuous [4], unlike in standard analysis; in paraconsistent set theory, the Russell set exists and is both in itself and not ${ }^{12}$, unlike in standard set theory; but

\footnotetext{
${ }^{11}$ For different presentations, see $[7,12]$ and references below.

${ }^{12}$ As in [38], also [6] in [3], also [5, ch. 8§4].
}

Australasian Journal of Logic (18:5) 2021, Article no. 14 
there is no conflict here. Priest encourages us to view these as sorts of language games, or just games. ${ }^{13}$ And all games are equally games. So, there is no sense in trying to de-legitimise one or another, or to view the existence of Go as a threat to Trivial Pursuit.

A conventional pluralist can try to absorb the apparently more radical pluralist, just by noting that what, say, a constructive mathematician is doing is in fact using a (classical) Heyting algebra, and a relevant mathematician is hanging their reasoning on (classical) Routley-Meyer frames ${ }^{14}$ : so it can all be viewed as just more (standard) mathematics. Now, the fact that one can translate one language into another does not mean that one can reduce one practice (carried out in that language) to another [26]. But once one puts on pluralistic spectacles, so to speak, apparently radical practices begin to seem not just legitimate, but not radical, because again it is all abstract structure in the void. Some arithmetics can be modeled with two elements, some cannot, and a thousand flowers etc.

What becomes of truth, according to a pluralist? For it is one thing to say that there are many formal geometries (indisputable), and another to ask which one is true. This appeal to truth might mean to ask which theory, say geometry, describes the actual world; or it might mean which formalizations correspond to our actual 'real' practice; or both; we return to this below. But a pluralist might try to steer around the issue altogether, and say that pluralism can perfectly well account for 'truth'. For example, Balaguer argues that "...there can be different theories of the same kind, that seem to be competitors, or incompatible, that are both true" [2], where by 'true' he means either a fictionalism about mathematics (in which case, they are all non-rivalrous stories) or plenitudinous platonism about mathematics

\footnotetext{
13 "There is a plurality of mathematical practices: category theory, intuitionist analysis and inconsistent calculus. Each of these is governed by a set of rules... and engaging in the practice means following the rules. The (institutional) point of following the rules is establishing (proving) certain - hopefully interesting - things within the rules of the practice. The rules may be explicit, as they typically are in contemporary mathematics; or implicit, as they were with number theory until the late 19th century. One may absorb the rules simply by being trained to follow them, as one learns a first language; arithmetic is usually learned in this way. Or one may learn the rules more reflectively, as one learns a second language; the way that a classically trained mathematician has to struggle with intuitionistic proof when they first meet intuitionist mathematics is like this. Just as with games, some practices may be more interesting, fruitful or whatever; but all practices, qua practices, are equally legitimate" [34].

${ }^{14}$ See [37].
}

Australasian Journal of Logic (18:5) 2021, Article no. 14 
(in which case, they are all describing different parts of platonic heaven and are again non-rivals). ${ }^{15}$ Truth, a pluralist can argue, is always relativised to some theory or practice, and on that view Meyer's work in relevant arithmetic is legitimate and valuable, but no challenge to the accepted wisdom about Gödel's theorems, which are about classical arithmetic. One can no more overturn the results of one mathematical practice via another than one could checkmate a chess opponent by outrunning them in a footrace. So on one pluralist reading, Meyer's results are interesting, but not revolutionary.

\subsection{A Monist View}

Mathematical monism I take to be a familiar and well-trodden view. The most common way to think of monism is as some version of mathematical Platonism, where there is a singular universe of mathematical objects 'out there' to be discovered, but the metaphysics don't matter much; monism could also be about mathematics as a deflated or nominalistic exercise which is nevertheless a unified practice. The key thing about monism, as I will understand it, is that unqualified mathematical questions have unambiguous answers (if they have answers at all), and mathematical singular terms have unambiguous meanings or referents. So the term 'the linear continuum' is not contextual, and neither is the question of whether it has $\aleph_{1}$ points or not: either it does, or it doesn't. According to monism, if you want to know whether there are infinitely many primes, it does not make sense to ask "According to what arithmetic'?" and it does make sense to ask, "Which formalization of arithmetic is correct?" The prevailing (but by no means necessary) view of monists ${ }^{16}$ is that something like ZFC set theory and its sub-theories like PA, all couched in classical logic, are mostly the correct formalization and foundation for all of mathematics.

A monist need not adhere to classical ZFC as a (singular) foundation, though. To see this, let's ask: how does relevant mathematics, and in particular Meyer's project, fare under a pluralist understanding? In terms of textual interpretation of the Great Relevant Literature and Meyer as a major exponent, pluralism would appear to be a misreading. Meyer does not

\footnotetext{
${ }^{15}$ Following the strategy from his book [1] where fictionalism and plenitudinous platonism are singled out as the best options for a philosophy of mathematics.

${ }^{16} \mathrm{~A}$ referee observes that graduate mathematicians today who are interested in foundations are often socially conditioned by their mentors, who are by and large steeped in classical orthodoxy.
}

Australasian Journal of Logic (18:5) 2021, Article no. 14 
phrase things in a pluralistic or relativistic way because he is concerned about 'intuitive' or true arithmetic, and he wants to claim that classical PA is not true arithmetic. Meyer sees formal logic as something imposed upon intuitive arithmetic, an attempt to interpret or describe the "logical superstructure" of the arithmetic facts. And for Meyer (as with contemporaneous Routley), PA has got the logical "superstructure" wrong. Real mathematics is not classical mathematics. ${ }^{17}$

A decent logic should avoid Gödel's theorems, Meyer seems to think; if a logic leads arithmetic into Gödel's theorems, this itself is a reason to doubt the logic as a good formal base for arithmetic. If a logic avoids Gödel, meanwhile, while still supporting 'natural' arithmetic reasoning, then this is a reason in favor of the logic. The Gödel "anomalies" are the result of a bad theory about logic, not a fact about arithmetic. The very fact that classical arithmetic is susceptible to Gödel "must certainly count as evidence" that there is something wrong with classical logic "as a rational approach to the formalization of arithmetic" [18, p. 26]!

This does not read as ecumenical pluralism. Different logical approaches are rivals; Gödel's theorems are a check on the success (or failure) of an attempted logical formalism. "What we wish to be sure of, as Hilbert might have put it, is that excursions through general logical laws...do not render dubious what we rightly regard as indubitable" [18, p. 11]. If a formalization of arithmetic cannot be shown to be reliable through elementary, finitary means that everyone can recognize, then it is not a successful formalization, because intuitive mathematics should admit of a formalization, on pain of mysticism. As he puts it,

From the present viewpoint, the task of furnishing a non-mythological and demonstrably secure reconstruction of all mathematics was interrupted over trivia, and it is time that these trivia were placed once more in proper perspective. Again, I do not propose to change the logical superstructure - only to understand it more clearly, by making explicit in a formal way features that have belonged to our intuitive logic all along [18, p. 11].

And as far as determining which formal logic captures the intuitive logic of arithmetic goes, it is no good to appeal to classical arithmetic to try to mediate this debate; that would be circular: "If we impose a certain

\footnotetext{
${ }^{17}$ This is emphasized at length in [38].
}

Australasian Journal of Logic (18:5) 2021, Article no. 14 
logic upon arithmetic, we cannot justify this imposition on the ground that arithmetic (among other things) respects this logic."

For Meyer, formalizing arithmetic well means distinguishing between descriptive facts, and constitutive laws. In terms of formalism, descriptive facts are 'extensional' and phrased in terms of $\wedge, \vee, \neg$, whereas laws are given in terms of the 'intensional' modal $\rightarrow$. Descriptions are mathematically stagnant, inert, whereas laws generate or underwrite the facts. For example, Meyer says that axiom A14

$$
\mathbf{s}(x) \neq 0 \vee 1=0
$$

that zero is not a successor (or else!), may be an arithmetic fact (in terms of a material conditional) but it is not a law (in terms of a relevant conditional), because it is negative and negation belongs to logic, which Meyer has already separated from arithmetic. ${ }^{18}$

So for Meyer, there is such a thing as True Arithmetic, and there are more or less successful attempts to theorize it. First there is natural reason and mathematical practice, on which "we are informally more or less agreed" [17, p. 6]. Then, like accountants come to check the books or grammarians sorting out the 'rules' of language (post hoc, of course, even if then ergo propter hoc) the logicians arrive to attempt to lend certainty to the indubitable, and are judged harshly if they fail. ${ }^{19}$

Priest's pluralism is consistent with this project, of finding the correct formalism for arithmetic: "There is a received practice, number theoretic reasoning, set theoretic reasoning or whatever. But there can be legitimate

\footnotetext{
${ }^{18}$ Similarly, Meyer expresses doubt about axiom A13, that if $x$ and $y$ have the same successor then they are equal, as the sort of thing that we should not need to say and which has a "collapsing effect" from the standpoint of $R^{\sharp}$.

${ }^{19}$ Here is how Meyer sees the connection between formal logic and rationality, with respect to the issue of logic itself: "I've heard enough of the widespread assumption that one cannot bring an objective rationality to bear on the claims of competing logics, presumptively because logic itself is involved in judging those claims ... Such pessimism is on a par with a view that, because we are components of physical systems, we cannot make rational judgments between competing physical theories. For we do not need logical theory to make us rational, any more than we need physical theory to make us physical objects. Logic is a product of our rationality, not its cause. While we may hope also that it will cause an improvement in the product, ... we can still stand outside [claims about logic], equipped with our everyday reason - fallible, error-prone, imprecise, self-interested, and even biased though it may be...." [19, p. 583].
}

Australasian Journal of Logic (18:5) 2021, Article no. 14 
disputes about what, exactly, the correct norms of that practice are." ${ }^{20}$ Qua Meyer, this makes the issue a dispute about how arithmetic is actually practiced by working mathematicians, and who, if anyone, gets it right.

\subsection{Ways to view Meyer's results}

Did Meyer overturn Gödel's second theorem? It looks like Meyer thought so; but here from the vantage of the 21st century, it looks like the considered interpretation of his work can break in at least the following ways.

Yes (monist) There is a true arithmetic, and relevant arithmetic is it; the classical formulation PA is not arithmetic. The fact that classical PA succumbs to Gödel's theorem only shows that it is not true arithmetic. True arithmetic, which is relevant, can prove its own reliability. Update as of 1992: there is good evidence that this means some propositions thought to be proven arithmetic theorems, like the quadratic residue formula, are not in fact truths of arithmetic. (They may be truths of some stronger (and hence less reliable) system.) Gödel's second theorem is repealed.

Yes (pluralist) 'Gödel's second theorem' is a non-rigid designator that is in fact relative to a particular system. There is no such thing as 'arithmetic' independent of some formal system, and so there is no absolute fact of the matter about whether or not Gödel's second theoremtaken unrestrictedly - is true. On this conception, Meyer successfuly established that arithmetic formulated relevantly can prove its own reliability in a way that classical PA cannot. There are two related results, Gödel-in-PA and Gödel-in- $R^{\sharp}$, and Meyer's result addresses the latter.

\footnotetext{
${ }^{20} \mathrm{He}$ goes on: "We formulate different sets of rules, trying to capture these. There can be a fact of the matter about who, if anyone, gets it right. In the same way, linguists can take a spoken language and try to formulate a set of rules which capture its grammar. Some grammars can just be wrong. Of course, once a set of formal rules is set up, they do characterize some language or other; and even if it is not the one targeted, it can still be spoken. Similarly, once rules for a mathematical practice are explicitly formulated, it can be followed. Thus, an advocate of paraconsistent set theory with unlimited comprehension does not have to claim that $\mathrm{ZF}(\mathrm{C})$ is wrong. $\mathrm{ZF}(\mathrm{C})$ is just as good a practice (qua practice) as paraconsistent set theory. It is just that those who adhere to it are wrong if they claim that it correctly characterizes our naive practice about sets" [34, p. 10].
}

Australasian Journal of Logic (18:5) 2021, Article no. 14 
No (monist) There is a true arithmetic, but relevant arithmetic is not it. Meyer proved that there is a formal system that has a finitary nontriviality proof, but that formal system is not arithmetic. Indeed, any theory with a finite model cannot be a theory of arithmetic, for that very reason. The monist here can be agnostic as to whether classical PA (or some second order variant) is true arithmetic or not, and can leave as an open question whether or not Gödel's second theorem holds for true arithmetic. They just need to hold that there is some absolute notion of being able to prove the reliability of arithmetic by finititary means, and that Meyer's proof does not address that notion. Such a monist may even dispute that Meyer's result holds, because it uses classical model theory and this monist is non-classical (see below).

No (pluralist) 'Gödel's second theorem' is a non-rigid designator that is relative to a particular system, and in fact its meaning in different systems (especially when those systems are couched in different languages) may so radically vary as to be incommensurable. The very fact that Meyer can use 'arithmetic' to refer to a system with a finite model, or that classical logicians can use 'arithmetic' to refer to a system with Löb's theorem, shows they are talking about such different things as to make any shared meaning impossible. So Meyer did not overturn Gödel's second theorem because there is no such thing to overturn. There is just Gödel-in- $L$ for different values of $L$, and the fact that the words look the same should not mislead us into thinking there is some overlap, any more than the homophones 'bank' (as in river) and 'bank' (as in financial) overlap.

Note that the monist position need not be the same as a classical position; one can be a relevantist monist. Note too that the two pluralist positions are very similar, except that the first maintains some room for communication between different parties, while the latter is persuaded by Quinean concerns about the inscrutability of reference and sees no justification for assuming we mean the same thing; changing the logic has changed the subject.

I am not chiefly concerned, in this paper, with textual interpretation or trying to discern through the dark glass of time what Robert K. Meyer actually thought about any of this, in 1976 or ever. I am not very able to discern what my contemporaries believe about things, let alone those who can no longer answer for themselves. Instead what I now plan to do is run 
through a few exercises in relevant/paraconsistent mathematics, now with these distinct above readings in the forefront of our minds, to see if any more clarity is gained once we see some non-classical mathematics in action. Calculemus.

\section{Relevant arithmetic in practice: theory and metatheory}

What does formalization of arithmetic with a relevant logic amount to? Meyer, and possibly others, seemed to think that a shift to relevant/paraconsistent logic for the practice of mathematics would be, on the whole, relatively painless - that the ordinary proofs we teach and use would on the whole survive unchanged when logic is reformulated from, let's say, classical to relevant. ${ }^{21}$ But Routley said to Meyer (according to Meyer) that perhaps the project of "a 'minimal mutilation' of the orthodox standpoint is too conservative; perhaps classical logic undermines classical mathematics more thoroughly than I have undertaken here to believe..." [18, p. 29]. I think that is correct - that a relevant (or just substructural paraconsistent) formulation of arithmetic, or its metatheory, is not going to be a matter of some minor aesthetic tweaks to the more classical versions - and in this section I will give some evidence why.

Here are two concrete examples of 'real' relevant arithmetic in action. I will approach these examples from the standpoint of a 'pure' relevant mathematician reasoning as informally and intuitively about schoolroom arithmetic as possible. One example is from elementary number theory, about prime numbers, and one is from metatheory, about models for relevant arithmetic. Both examples bring out the effect of using a relevant/paraconsistent logical superstructure. ${ }^{22}$ A third example is relegated to the appendix.

\footnotetext{
21 "[I]f we are to think relevantly about mathematics, what is to be hoped for most of all are not new routes to old truths but an expansion of the pragmatic imagination" [17, p. 5].

${ }^{22}$ Invaluable sources on working within substructural arithmetic 'in the wild', are $[10$, 40], [35, ch. 11].
}

Australasian Journal of Logic (18:5) 2021, Article no. 14 


\subsection{Prime numbers in relevant arithmetic}

As you know, a number is prime iff it is only divisible without remainder by itself. ${ }^{23}$ Let us try to formalize this in $R^{\sharp}$, in two different ways, for two different results. Let $\mathbf{s} x$ be the successor of $x$ and $\times$ be multiplication, with standard properties (cf. [17, pp.92-97]). Let $\operatorname{div}(x, y)$ mean that $x$ divides $y$ without remainder: that $\exists n(x \times n=y)$ for $n \in \mathbb{N}$.

\subsubsection{Option 1: As a logical law}

Let us say that a number $p>1$ is prime iff

$$
\forall x(\operatorname{div}(\mathbf{s s} x, p) \rightarrow \mathbf{s s} x=p)
$$

This looks relatively promising; it treats being prime as a logical law (following Meyer's distinction) and reads as "for $p$ greater than $1, p$ is prime iff to divide $p$ is just to be $p . "{ }^{24}$ Actually this is a very strong statement when stated with $\rightarrow$, as Dunn explored with such relevant identity statements [8], but then, prime numbers are very strong so maybe that's okay.

Okay, that is, until one asks, what about composite numbers? A composite number is one that can be divided: a number $n>1$ is composite iff $\exists x(\operatorname{div}(\mathbf{s} \mathbf{s} x, n) \wedge \mathbf{s s} x \neq n)$. If that is taken as the definition of 'composite' then we appear to lose the fact that every number greater than 1 is either prime or composite. This is because $\neg(A \rightarrow B)$ does not entail $A \wedge \neg B$, and neither does $\neg(A \wedge \neg B)$ entail $A \rightarrow B$. So the familiar duality fails. If a number $p$ is not prime, then $\exists x \neg(\operatorname{div}(\mathbf{s s} x, p) \rightarrow \mathbf{s s} x=p)$, but that's all one can say; negated conditionals are an inferential dead-end in relevant logic. And if a number is not composite, then $\forall x(\neg \operatorname{div}(\mathbf{s s} x, n) \vee \mathbf{s s} x=n)$, but that

\footnotetext{
${ }^{23}$ But wait - that makes 1 a prime number, as Aristotle would have approved. For reasons of nicety, it is better for 1 not to be prime. So a number is prime is it is not divisible without remainder by any numbers other than 1 or itself. So the definition of prime number is negotiable, even before getting to logical issues; cf. [15]. For brief comments on prime numbers by Meyer, see [18, p. 63].

${ }^{24}$ The trick of using the successor of $x$ is inspired by Slaney in [40]. It avoids the need for caveats about 0 and 1 , or

$$
\forall x(x>0 \wedge \operatorname{div}(x, p) \rightarrow x=p \vee x=1)
$$

which is handy because otherwise there is an extra disjunct in the consequent, and the contrapositive ends up as $x \neq p \wedge x \neq 1 \rightarrow \neg(x>0) \vee \neg(\operatorname{div}(x, p))$. Disjunctions become cumbrous without disjunctive syllogism.
}

Australasian Journal of Logic (18:5) 2021, Article no. 14 
won't get us up to being prime without disjunctive syllogism. Of course, one could simply define composite numbers as those which are not prime, and regain that every number is either prime or composite, but at the cost of being able to use composite numbers for much of anything, because they are defined as negated conditionals.

\subsubsection{Option 2: As a descriptive fact}

Let us say that a number $p>1$ is prime iff

$$
\forall x(\neg \operatorname{div}(\mathbf{s s} x, p) \vee \mathbf{s s} x=p)
$$

Then it would be quite natural to say that a number $n>1$ is composite iff $\exists x(\operatorname{div}(\mathbf{s s} x, n) \wedge \mathbf{s s} x \neq n)$. Doing so, we find immediately that every number greater than 1 is either prime or composite by the duality of conjunction/disjunction and existential/universal quantifiers, and the law of excluded middle. Excellent.

This looks even more promising than the somewhat over-exuberant attribution of lawmaker status to primes .... more promising, that is, until one asks, what about the prime factorization theorem (that every number has a unique set of prime factors)? One surely expects 'true' arithmetic to deliver that. Euclid could deliver that by etching diagrams in sand. ${ }^{25}$ What about a stepping stone to that fundamental theorem, Euclid's 'first theorem', which says that if a prime number $p$ divides a product $x \times y$, then either $p$ divides $x$ or $p$ divides $y$ ? Well, you are probably better at proving things than me, but even arguing in a relatively natural and flexible way (which you can't really do in $R^{\sharp}$ ), starting from the extensional definition of composites then all I get is

If $p$ is prime and $\operatorname{div}(p, x y)$, then $\operatorname{div}(p, x)$ or $\operatorname{div}(p, y)$, or $p \neq p$.

Proof: Assume $p$ divides $x y$. By linear order, where $n<m \vee m \leqslant n$ (see [17, p. 112]), either the greatest common divisor of $p$ and $x, \operatorname{gcd}(p, x)$, is greater than 1 , or identical to 1 . (Let's suppose that if it were 0 , that would be absurd (whence anything, including the result, follows).) If $\operatorname{gcd}(p, x)=1$,

\footnotetext{
${ }^{25}$ Or probably wax, since sand would get too messy [29].
} 
then there exist $u, v$ such that

$$
1=p u+x v
$$

Therefore

$$
y=p u y+x v y
$$

by multiplying $y$ to both sides. But since $p$ divides $p u$, and $p$ divides $x y$ on assumption, $p$ must divide both puy and $x v y$. Then $p$ also divides the sum $p u y+x v y=y$. Super. But if $\operatorname{gcd}(p, x)>1$ then by the definition of $p$ being prime, then either $\operatorname{gcd}(p, x)=p$-whence $p$ divides $x$, which is good - or else $p$ does not divide $p$, and so $p 1 \neq p$. Well, it would be funny if $p 1 \neq p$, but we can't rule it out if we are aiming for a paraconsistent arithmetic. So the proof stops there with that case unresolved.

Thus using the 'descriptive' material conditional phrasing leaves us with some residual possibility of inconsistency that cannot be eliminated. This residual possibility follows us right along, through the standard sequence of proofs all the way to the

[Fundamental Theorem of Arithmetic] Let $n>1$. Then there are primes $p_{0}, \ldots, p_{m}$ such that

$$
n=\prod_{i=0}^{m} p_{i}
$$

and for any other such $q_{0}, \ldots, q_{\ell}$, either each $p_{i}$ is identical to exactly one $q_{j}$ ... or some $p_{i} \neq p_{i}$.

So one gets 'uniqueness up to inconsistency': if $p \neq p \rightarrow \perp$ for prime $p$, then prime factorization is unique; but if not, not. ${ }^{26}$

The moral here is that substructural arithmetics are very sensitive to the way notions are formulated, and classically equivalent ways are not equivalent. The negation connective has been designed to permit more possibilities, an expansion of the imagination - "Let us be free to wonder what it would be like if 0 were equal to 2 , and let us not be stopped short by our conviction that 0 isn't 2" [17, p. 5] - but in doing so the old routes to old truths are obscured. If there is a single true notion of prime number, it is unclear how to isolate it, except to see how various formulations fare. So the monist must ask the real prime numbers to please stand up. The pluralist meantime sees many different prime number games to play.

\footnotetext{
${ }^{26}$ For details, see [41, ch. 6].
}

Australasian Journal of Logic (18:5) 2021, Article no. 14 


\subsection{Is relevant arithmetic incomplete with respect to classical arithmetic?}

We've just had an example of the complications that arise in doing basic number theory in $R^{\sharp}$ and related logics. Now lets see what happens when we try to study $R^{\sharp}$ itself, in a mathematically precise way. We revisit the seminal "Whither relevant arithmetic?" of 1992, wherein Friedman and Meyer prove a result that they presented as saying that $R^{\sharp}$ will not 'recapture' all of classical PA [11] . A shift to 'relevant metatheory' does not quite repeal this result, but it does undermine the result, as I will sketch. ${ }^{27}$ Our various monist/pluralist readings of Meyer's Gödel result can be applied to this example, too.

Friedman and Meyer answer the open question of the admissibility of gamma, in the negative. This was the general question of whether $R^{\sharp}$ could do everything classical arithmetic can do, via the specific question of whether there are any theorems obtainable with disjunctive syllogism not obtainable without it. They answer the question by showing that there is a theorem of PA that is not a theorem of $R^{\sharp}{ }^{28}$

I will sketch the Meyer-Friedman argument, and flag where substructurally invalid steps are used (apparently essentially), with a '!'. The argument proceeds by forming a classical theory $P^{+}$: (1) Form $R^{\sharp+}$ as all the positive theorems (with arrow but without negation) of $R^{\sharp}$, and so without the axiom ' 0 is not the successor of any number' (since that has a 'not' in it); (2) Get $P A^{+}$by adding $A \rightarrow(B \rightarrow A)$ and $A \vee(A \rightarrow B)$; (3) Definitionally extend to $P^{+}$by defining negation as

$$
\neg A:=(A \rightarrow 0=1)
$$

and adding

0 is not the successor of 0

which given the definition of negation is the axiom $0=1 \rightarrow 0=1$, which is already a theorem. Then all strictly positive theorems of $R^{\sharp}$ are theorems of $P^{+}$. It is already known (via a classical argument) that $R^{\sharp}$ is a conservative extension of its positive fragment (see, e.g. [27].

\footnotetext{
${ }^{27}$ Meyer discusses the issue of a 'relevant metatheory' at length in [17] and also later in $[20]$.

${ }^{28}$ This result can be obtained (classically), basically, because relevant arithmetic is flexible, as the existence of finite models already show. Meyer and Urbas [27] point out that easy "conservative extension results are characteristic of many relevant theories" in part because relevant theories have some pretty non-standard models. Indeed.
}

Australasian Journal of Logic (18:5) 2021, Article no. 14 
Rule $\gamma$ (detachment for $\supset$ ) is admissible iff adding it as a rule does not change the set of theorems. This seems required, if $R^{\sharp}$ is to be as strong as PA. If $\gamma$ is admissible in $R^{\sharp}$, then the following purely classical condition is true: every strictly positive theorem of $P A$ is already a theorem of $P^{+}$. Call this the fundamental condition (FC). But the $\mathrm{FC}$ is false. Thus, by (a very dubiously valid !) contraposition, $\gamma$ is not admissible in $R^{\sharp}$. To see this, we look at the Complex Ring $\mathbb{C}$, and show it is a model of $P^{+}$.

A set $\alpha$ is definable when it satisfies some formula $A$. A set is cofinite when its complement is finite. Let $\mathbb{C}$ be the ring of complex integers. Friedman and Meyer prove that every definable subset of $\mathbb{C}$ is either finite or cofinite. ${ }^{29}$ Then $\mathbb{C}$ is a model of $R^{\sharp}$, if all the axioms of $R^{\sharp}$, including the following "powerful" form of induction, hold in it $[11$, p. 828]:30

$$
\exists x A x \wedge \forall x(A x \rightarrow A s x) \rightarrow \forall x A x
$$

Proof of induction in $\mathbb{C}$ : Assume the antecedent $\exists x A x \wedge \forall x(A x \rightarrow A \mathbf{s} x)$ is true, and let $\alpha$ be the subset of $\mathbb{C}$ for which $A$ holds. Then $\alpha$ is not finite (!), so it is cofinite (disjunctive syllogism !); but then $\mathbb{C} \backslash \alpha$ cannot be cofinite (proof by contradiction !); since on assumption $\mathbb{C} \backslash \alpha$ is closed under predecessor, it must be empty (proof by contradiction !). Ergo $\alpha=\mathbb{C}$ (because if $X \backslash Y=\emptyset$ then $X=Y$ !).

A check of the other axioms and rules shows that $\mathbb{C}$ is a model of $P^{+}$. But the quadratic residue formula (QRF) - for every odd $n$ there is an integer that is not a quadratic residue $\bmod n-$ is a theorem of $P A$, and not a theorem of $P^{+}$, because it is false in $\mathbb{C}$, which is a model of $P^{+}$. Then QRF is not a theorem of $R^{\sharp}$. Therefore FC fails for $P^{+}$. Therefore, we are told to conclude that relevant arithmetic is $P A$-incomplete and gamma fails for it.

Does our view of the Meyer-Friedman result change if we work in purely relevant theory and metatheory? The proof that $\mathbb{C}$ models $R^{\sharp}$ is very classical. The authors are explicit about this - "it's a purely classical question!", they say, published in the very classical Journal of Symbolic Logic, so I don't mean

\footnotetext{
${ }^{29}$ The proof requires the fundamental theorem of algebra, where an equation of degree $n$ has at most $n$ roots in $\mathbb{C}$. It is highly dubious (!) that this can be paraconsistently recaptured as stated.

${ }^{30}$ This is the form given in Friedman and Meyer's paper. As a referee points out, the $\exists$ in the antecedent is funny as the base case for the induction, since e.g. 6 is greater than 5 , and for all numbers greater than 5 their successors are too, but not all numbers are greater than 5. My guess is that Friedman and Meyer are showing that this is indeed a "surprising" model, which overgenerates (and still comes up short).
}

Australasian Journal of Logic (18:5) 2021, Article no. 14 
to be criticizing the proof as if Meyer made some kind of mistake here. But the proof, if it is intended to be persuasive, does assume that the "logical superstructure" of metatheory (the study of what theorems belong to what theories) is classical. This proof for the non-admissibility of gamma would not survive unmutilated into a relevant reformulation of mathematics. It assumes that the algebra of sets (e.g. subsets of $\mathbb{C}$ ) is boolean and uses disjunctive syllogism essentially. More, the proof that $\mathbb{C}$ is a model of $P^{+}$ assumes that a subset in which $\exists x A x \wedge \forall x(A x \rightarrow A x+1)$ holds cannot be finite. Can’t it? Have we learned nothing from the finite models of $R^{\sharp}$ ?!

Without this last step especially, the whole 'Whither' argument breaks down. It is not at all clear to me that, from the standpoint of a committed relevant/paraconsistent mathematician, one should take this result as dispositive. I think a (very) committed relevant number theorist could, at present, say that the status of the Quadratic Residue Formula in relevant arithmetic remains open. From the standpoint of relevant mathematics (alone), the adequacy of relevant arithmetic remains undetermined. ${ }^{31}$

\subsection{Is relevant arithmetic arithmetic?}

The above excursions illustratively suggest, I think, that the choice of formalism - imposing a classical versus relevant superstructure - is much more than a superficial choice between fashion accessories. The 'not-logic' part of arithmetic (and mathematics) Meyer presumes is harder and harder to see. In the decades since the 1970s, it has become apparent that relevant/paraconsistent mathematics cannot simply absorb standard orthodox mathematics by making a few minor adjustments. ${ }^{32}$ The differences are too pronounced. This isn't to say that the majority of informal mathematics is classical. But it isn't straightforwardly relevant either. So we are thrown back to the pluralism versus monism question, of how to understand Meyer's

\footnotetext{
${ }^{31}$ What about the admissibility of gamma itself? This depends on how likely one thinks that any inconsistencies in arithmetic are actually forthcoming, as Priest has argued, versus being mere possibilities, as Meyer suggests. (Cf. Appendix below.) I'd say that hopes for keeping all the consequences of disjunctive syllogism while still being paraconsistent are not merely utopian, but deeply misguided...but rather than argue that, I'll just ask: would Meyer class gamma as a 'descriptive fact' or a logical law? (Cf. [19].) It seems like it would be a fact; he is hoping it is just out there in the arithmetic topography, admissible without being needed. But this leaves open still whether gamma is even true.

${ }^{32}$ Contra plans outlined in e.g. [38].
}

Australasian Journal of Logic (18:5) 2021, Article no. 14 
work, and indeed how to understand paraconsistent arithmetic(s) and mathematics in general.

Is relevant arithmetic arithmetic? Well, a toy duck is not a duck, so just calling something "relevant arithmetic" does not arithmetic make. If monism is correct, then either $R^{\sharp}$ (or some variant) is arithmetic or it isn't. And then it's a matter of what you mean by 'arithmetic', which I'll return to in the conclusion. But given that different formalizations - precisifications - of our informal talk about e.g. primes tend in very different directions, and different inferential norms about e.g. models tend too to give different answers about the adequacy of $R^{\sharp}$, these exercises might suggest a push toward pluralism instead - just different ways to do different things.

I'm not sure we should be so quickly satisfied, though. A toy duck is not a duck. And to push a strong version of the pluralist line, there is no such thing as unqualified arithmetic, without some relativization. There are only different toy ducks, of various sorts, and no ducks simpliciter at all. No ducks? Having reached this alarming question, it is time to conclude.

\section{Conclusion: Thither (relevant) arithmetic!}

A fair verdict here might be to say that Meyer's argument (or rhetoric) reads as monistic, while his results today look primed for a pluralistic reading. Is there a way to thread this needle?

It depends on targeting exactly what we mean by pluralism $\mathrm{v}$ monism about mathematics. Let's assume that Meyer is mainly concerned with mathematical practice, how mathematicians talk and prove in ordinary informal contexts. (This is as opposed to concern with outright platonistic mathematical truth, which is a matter of what objects exist and what properties they have, whether we talk about them or not.) As we've seen, first there is the informal mathematizing at the whiteboard, which Meyer and we believe has a logical superstructure; then there is the formalization thereof. But the formalization may serve two different purposes. One is descriptive, a matter of recording which canons of reasoning people actually seem to use, even implicitly. Another is normative, offering adjustment and correction to show which cannons of reasoning people ought to be using. The question of pluralism v monism about Meyer's Big Claim can be sharpened, if not answered, by filtering it through this distinction.

For a relevant logician, a monism centered on description of mathemat-

Australasian Journal of Logic (18:5) 2021, Article no. 14 
ical practice does not look very promising; cf. [32, p. 221]. If Meyer's Big Claim is that actual ordinary arithmetic as it is taught and used by Everyday Working Families is better formulated using a relevant logic, then he is probably wrong. If the project is to describe (roughly) what we find schoolteachers, or Terry Tao et al, working with to actually prove things, I doubt it is paraconsistent, let alone $R$ or a nearby logic. (Arguably, I would even say $R$ is too stringent to fully describe the norms people actually (would like to) believe in.) Now, a pluralist could try to slow things down here and say that there is no 'one' thing people are doing called mathematics, that if there are (is?) paraconsistent set theorists then paraconsistent logic does correctly describe that behaviour; and if Meyer was a relevant arithmetician then relevant arithmetic describes him. But Meyer was not discussing mathematics as practiced by a small cadre of logicians in Australia ${ }^{33}$; Meyer meant 'ordinary' mathematics. And while I don't concede that classical logic is the right description of ordinary mathematical practice, I would concede that, as a description, relevant logic is not either.

So maybe a free-to-be-you-and-me pluralism is the better way forward, for relevant mathematics. In one of his late publications, Meyer states that there is no One True Logic, not even One True Relevant Logic. There are too many coherent variations on the Routley-Meyer semantics that dial the strength of the logic up or down so finely that there is little hope of discerning the exact right setting. ${ }^{34}$ If there is no one true logic, the pluralist approach is looking better after all.

But there is still a straw to grasp at for the monist. It is the normative straw. Meyer's critical argument against classical arithmetic is about negation, and while he appeals to how 'not' actually works in our actual language, I submit that he is more convincingly appealing to how negation ought to work (or how it does work, in the Mind of God). An adequate and reliable theory of arithmetic will have a negation that commutes transparently with its provability (and truth) predicates, not because that's actually how things are, but because that's how things ought to be. Meyer's claim looks revi-

\footnotetext{
${ }^{33}$ See [39], which puts things more or less just this way.

34 "The present overview ... makes clear (despite some of our hopes and utterances) that the One True Logic does not exist [emphasis original]. ...[S]ubtle and not so subtle variations on semantical postulates produce different logics in the same family. The question of which semantical postulates are correct makes no sense without further context" [16, p. 280]. Note the first author on this article is Mares, who I gather took main responsibility for the parts of the article that aren't jokes.
}

Australasian Journal of Logic (18:5) 2021, Article no. 14 
sionary because it is revisionary: on this reading, anyone not formalizing mathematics with a paraconsistent negation (and for now, that might be $e v$ eryone) is doing something wrong. Gödel's theorems may be based on dirty tricks, but they are tricks we've all been falling for. To improve, we all need to start walking together out, toward the light.

On this normative approach, if True Arithmetic's negation must respect the behavior of negation - not necessarily in our language, but in Plato's ideal sky - then such negation may yet be paraconsistent, and then so would be True Arithmetic. If relevant or paraconsistent logic is a corrective to our practices, then the fact that our practices have not yet been so corrected is irrelevant; it means there is more to do. And then, maybe, Meyer was right, and Gödel's theorem isn't the end of the story. But for now, this is: Whither relevant arithmetic? Thither relevant arithmetic.

\section{Appendix: Is gamma even admissible in $R^{\sharp}$ ?}

With the (apparent) demise of $R^{\sharp}$ as a replacement for classical $P A$, some got to wondering whether a slightly stronger system could fit the bill-something in which gamma is admissibile, but still escapes Gödel. The system $R^{\sharp \sharp}$ is obtained by adding an $\omega$-rule:

omega rule If $\vdash A(n)$ for every numeral $n$ then $\vdash \forall x A(x)$.

(Individual variables may not appear free in theorems at all.) Meyer argued that $R^{\sharp \sharp}$ is PA complete after all. Then, since most people think the omega rule is a rule the way 'Do it correctly' is helpful advice, there has been some hope of an R-sharp-and-a-half, that somehow (again) hits the sweet spot: $R^{\sharp 1 / 2}$ is weaker than $R^{\sharp \sharp}$, in that it has a less magical rule than the omegarule, but it is stronger than $R^{\sharp}$ in that it can prove things like the quadratic residue formula. ${ }^{35}$ To my knowledge, this unicorn remains undiscovered. But, Meyer consoled us, at least we know that there is a system, $R^{\sharp}$, that is true arithmetic [22].

In the spirit of the above exercises in substructural mathematics and metatheory, I'd like to gently challenge even the result that gamma is admis-

\footnotetext{
${ }^{35} \mathrm{~A}$ referee points out that the precise form of induction of any kind is a very sensitive issue in virtually every formalisation of arithmetic, including type-theoretic and categorytheoretic formulations.
}

Australasian Journal of Logic (18:5) 2021, Article no. 14 
sible in $R^{\sharp \sharp}{ }^{36}$ I will again mark paraconsistently dubious steps with '!'. In $[22$, p. 7] Meyer proves what he calls the truth theorem:

If $\mathrm{A}$ is true in the standard model of arithmetic, then $\vdash A$ in $R^{\sharp \sharp}$.

I'll take this as proved, though the argument involves steps like adding as an axiom that $t=u$ whenever $t=u$ is a correct numerical equation. (A numerical equation is correct when "it is so according to the algorithms for addition and multiplication that we all learned in school" [22, p. 6].) Assuming the truth theorem, how is the admissibility of gamma proved? The proof is a reductio: suppose that $A \supset B$ and $A$ are theorems, but $B$ is not. Show (via an argument due to Belnap) that there is an extension $T$ of $R^{\sharp \sharp}$ where $B$ is still not a theorem, and there is a 'really rotten countertheory $F$ ' of sentences that are not in $T$. This needs to be further refined, though, since $T$ may be negation-inconsistent (it may include theorems and their negations) in which case there is no hope of gamma. So further refine $T$ to a consistent subtheory (!) $T R$ containing $R^{\sharp \sharp}$, where $\neg A \in T R$ iff $A \notin T R$. Then gamma is admissible. For suppose $A \in T R$ and $\neg A \vee B \in T R$ but $B \notin T R$. By construction, $\neg B \in T R$. Then $A \& \neg B \in T R$. Then by de Morgan rules, $\neg(\neg A \vee B) \in T R$, so $T R$ is negation inconsistent, which is a contradiction. Thus $B \in T R$, and gamma is admissible (!!!).

So, this is a classical proof by contradiction, with almost all of its working parts turning on the consistency of the underlying metatheory. It proves gamma is admissible, by using disjunctive syllogism in the argument, and so a tacit assumption of consistency. For $T R$ is only consistent if the condition defining it is: if it is possible that some $C \in T R$ and $C \notin T R$ then there proof stops. This point is not lost on Meyer. In a footnote following the 'truth theorem', (where he suggests that Gödel's first incompleteness theorem actually proves a contradiction (cf. $[32,17 \S 5])$ ) ) he says (boldface original):

There is, in all of this, a generous helping of the Standard Mythology. It may be that we are confused about truth in the standard model $\mathbb{N}$ of the natural numbers. One could demonstrate this simply by improving Gödel to the point where an actual proof of $A \& \neg A$ for some $A$ is classically forthcoming (in, say, first-order

\footnotetext{
${ }^{36}$ I'd not be the first to cast some doubt, if not on the details then on the importance of the result. According to [25, p. 1], Restall disputes that even arr-sharp-sharp is true relevant arithmetic. "'How can that be,' he wanted to know, 'when there are sentences $A$ of $R^{\sharp \sharp}$ such that neither $A$ nor $\sim A$ is a theorem?' An example is $0=2 \rightarrow 0=1$."
}

Australasian Journal of Logic (18:5) 2021, Article no. 14 
Peano arithmetic). But, for present purposes, we are content to suppose not; whence exactly one of $A, \neg A$ is true in $\mathbb{N}$, for each arithmetical sentence $A$ [22, p. 349].

Supposing consistency may be good enough for "present purposes". The future awaits.

\section{Acknowledgments}

My sincere thanks to Thomas Ferguson and Graham Priest for the opportunity to write this paper. Versions of it were presented at the New Zealand Association of Philosophers Conference 2020 and at the University of Otago in 2021. Material in Section 4.2 was originally presented as "Thither Relevant Arithmetic!" back in 2013, at UniLog 4 in Rio de Janiero and the Australasian Association of Logic in Melbourne. Thanks to all those audiences, and a very helpful anonymous referee at the AJL, and Patrick Girard, for valuable comments.

\section{References}

[1] M. Balaguer. Platonism and Anti-Platonism in Mathematics. Oxford University Press, 1998.

[2] M. Balaguer. Mathematical pluralism and platonism. Journal of Indian Council of Philosophical Research, 34:379-398, 2017.

[3] D. Batens, C. Mortensen, G. Priest, and J.-P. van Bendegem, editors. Frontiers of Paraconsistent Logic. Research Studies Press, Philadelphia, 2000.

[4] J. L. Bell. A Primer of Infinitesimal Analysis. Cambridge University Press, 2008. Second Edition.

[5] W. Carnielli and M. E. Coniglio. Paraconsistent Logic: Consistency, Contradiction and Negation. Springer, 2016.

[6] N. da Costa. Paraconsistent mathematics. In Batens et al. [3], pages 165-180.

[7] E. B. Davies. A defense of mathematical pluralism. Philosophia Mathematica, (13):252-276, 2005.

[8] J. Dunn. Relevant predication 1: The formal theory. Journal of Philosophical Logic, 16:347 - 381, 1987.

Australasian Journal of Logic (18:5) 2021, Article no. 14 
[9] J. M. Dunn. A theorem in 3-valued model theory with connections to number theory, type theory, and relevant logic. Studia Logica, 38:149$169,1979$.

[10] J. M. Dunn. Relevant robinson's arithmetic. Studia Logica, 38:407-418, 1980.

[11] H. Friedman and R. Meyer. Whither relevant arithmetic? Journal of Symbolic Logic, 57:824-31, 1992.

[12] M. Friend. Pluralism in Mathematics: A New Position in Philosophy of Mathematics. Springer, Dordrecht, 2013.

[13] J. D. Hamkins. The set-theoretic multiverse. Review of Symbolic Logic, 5:416-449, 2012.

[14] W. Hodges. An editor recalls some hopeless papers. Bulletin of Symbolic Logic, 4(1):1-16, 1998.

[15] I. Lakatos. Proofs and Refutations: The Logic of Mathematical Discovery. Cambridge University Press, 1976.

[16] E. D. Mares and R. K. Meyer. Relevant logics. In L. Goble, editor, Blackwell Guide to Philosophical Logic, pages 280-308. Blackwell, 2001.

[17] R. K. Meyer. Arithmetic formulated relevantly. typescript, 1975.

[18] R. K. Meyer. The consistency of arithmetic. typescript, 1976.

[19] R. K. Meyer. A farewell to entailment. In G. D. et al, editor, Foundations of Logic and Linguistics, pages 577-636. Springer Science and Business Media, New York, 1985.

[20] R. K. Meyer. Proving semantical completeness "relevantly" for R. Logic Research Paper, (23), 1985. RSSS Australian National University.

[21] R. K. Meyer. Kurt Gödel and the consistency of $R^{\# \#}$. In P. Hájek, editor, Gödel '96: Logical Foundations of Mathematics, Computer Science and Physics - Kurt Gödel's Legacy, Brno, Czech Republic, August 1996, proceedings, pages 247-256. Springer-Verlag, Berlin, 1996.

[22] R. K. Meyer. つ-E is admissible in 'true' relevant arithmetic. Journal of Philosophical Logic, 27:327-351, 1998.

[23] R. K. Meyer and C. Mortensen. Inconsistent models for relevant arithmetics. Journal of Symbolic Logic, 49:917-929, 1984.

[24] R. K. Meyer and C. Mortensen. Alien intruders in relevant arithmetic. Technical Report, TR-ARP(9/87), 1987.

[25] R. K. Meyer and G. Restall. 'Strenge' arithmetics. Logique et Analyse, 42:205-220, 1999.

[26] R. K. Meyer and R. Routley. Extensional reduction (i). The Monist, 60:355 - 369, 1977.

Australasian Journal of Logic (18:5) 2021, Article no. 14 
[27] R. K. Meyer and I. Urbas. Conservative extension in relevant arithmetic. Mathematical Logic Quarterly, 32(1-5):45-50, 1986.

[28] C. Mortensen. Inconsistent Mathematics. Kluwer Academic Publishers, Dordrecht; New York, 1995.

[29] R. Netz. The Shaping of Deduction in Greek Mathematics. Cambridge University Press, 1999.

[30] G. Priest. Is arithmetic consistent? Mind, 103, 1994.

[31] G. Priest. Inconsistent models of arithmetic part I: Finite models. Journal of Philosophical Logic, 26:223 - 35, 1997.

[32] G. Priest. In Contradiction: A Study of the Transconsistent. Oxford University Press, Oxford, 2006. Second edition.

[33] G. Priest. An Introduction to Non-Classical Logic. Cambridge, 2008. Second Edition.

[34] G. Priest. Mathematical pluralism. Journal of the IGPL, 21:4-13, 2013.

[35] G. Restall. On Logics Without Contraction. PhD thesis, The University of Queensland, January 1994.

[36] G. Restall. Carnap's tolerance, meaning and logical pluralism. Journal of Philosophy, 99:426-443, 2002.

[37] G. Restall. Models for substructural arithmetics. Australasian Journal of Logic, 8:82-99, 2010.

[38] R. Routley. Ultralogic as universal? Relevance Logic Newsletter, 2:5189, 1977. New edition as The Sylvan Jungle vol. 4, edited by Z Weber. Synthese Library, 2019.

[39] S. Shapiro. Varieties of Logic. Oxford University Press, 2014.

[40] J. Slaney. The square root of two is irrational (and no funny business). typescript, 1982.

[41] Z. Weber. Paradoxes and Inconsistent Mathematics. Cambridge University Press, 2021. in process.

Australasian Journal of Logic (18:5) 2021, Article no. 14 